\title{
An Unusual Presentation of Lipoma on the Dorsum of the Foot in A 9 Year Old Girl: A Case Report and Review of the Literature
}

\author{
Agu Thaddeus Chika*
}

Consultant Surgeon and lecturer, college of medicine, Imo State University, Owerri, Nigeria

"Corresponding Author: Agu Thaddeus Chika, Consultant Surgeon and lecturer, college of medicine, Imo State University, Owerri, Nigeria, Tel: +234 8034053944; Email: tcagu@ yahoo.com

Received: 21 September 2017; Accepted: 28 September 2017; Published: 30 September 2017

\begin{abstract}
A lipoma can occur in virtually any organ of the body that has fat cells. A slow growing mass on the dorsum of the foot in a 9 year old girl which had recently ulcerated and was painful appeared sinister. In the absence of trauma or local scarification on the mass, the spontaneous denudation of the overlying skin and eventual ulceration with discharge of serous fluid seemingly made malignancy a probable but not a definite diagnosis. The groin nodes were enlarged and tender but there was no cough and no weight loss. Biopsy confirmed the diagnosis of lipoma. The aim of this report is to highlight the need for the clinician to be broad minded in the management of patients and to consider the psychological impact of making a pronouncement without a confirmatory diagnosis.
\end{abstract}

Keywords: Lipoma; Unusual presentation; Psychological impacts; Biopsy

\section{Introduction}

Lipoma is a benign neoplasia of adipocytes consisting of mature fat cells in a thin fibrous capsule. It is the commonest form of benign soft tissue tumor of mesenchymal origin. Lipoma is regarded as a 'ubiquitous' tumor or 'universal' tumor [1, 2]. Solitary lipomas are uncommon in children and they affect more women than men between the ages of 40 and 60 years [1-3]. Lipomas mainly occur in the head and neck and shoulder regions as well as proximal extremities [3]. The fat cells in this lesion are rapidly dividing but are still within their confines, and also maintain their cell morphology- in the aspects of shape, size, uniformity, tissue boundary and nucleo-cytoplasmic ratio on microscopy. The lesion therefore is most unlikely to ulcerate through the skin except there is a malignant 
transformation which is extremely rare [4] or there is trauma from frictions or ulceration following infected scarification wounds. A rapidly enlarging mass can exact a lot of pressure on the skin, causes local ischemia, necrosis, sloughing and ulceration. Benign masses are not known to commonly grow very rapidly and so the investing structures have time to expand to accommodate it. Though a mass may appear to be associated with some clinical features of malignancy, it should not be pronounced malignant until a complete diagnostic work up is done. There is no literature on this kind of unusual presentation of lipoma on the distal extremity in a child especially with ulceration. This report highlights the difficulty with clinical diagnosis of an uncommon presentation of lipoma and the need to avoid avoid psychological trauma on the patient and family members by doing a complete diagnostic work-up before a definitive pronouncement.

\section{Presentation of Case}

A 9 year old girl presented to us with an initially painless swelling on the dorsum of the left foot of 19 months (Figures 1 and 2). The mass was increasing in size gradually until the past 5 months when the patient noticed rapid increase in its size and pain in the foot. The mass was disturbing her ability to put on her shoes. There was no weight loss. Four weeks prior to presentation, the mass ulcerated and was discharging serous foul smelling fluid (Figure1). She said that there was no form of trauma or scarification on the mass. The patient had already been seen by their family doctor who after assessment pronounced that she may be having cancer and probably would need amputation. In great distress, patient's mother brought her to our level II surgical facility for second opinion.

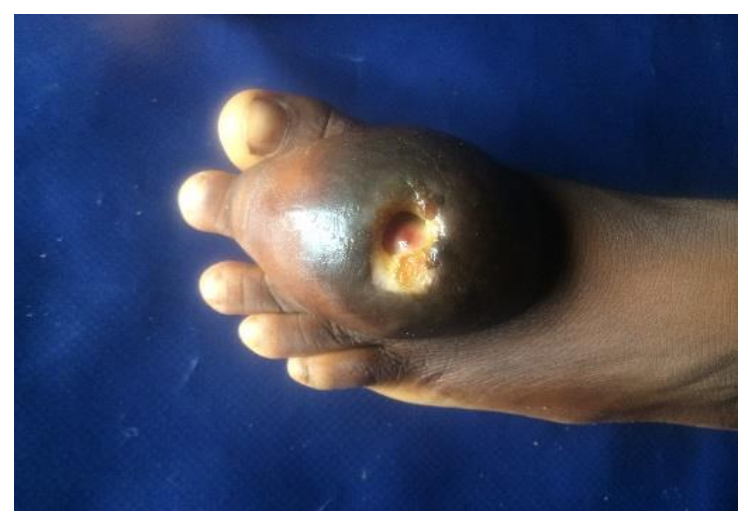

Figure 1: A clinical photograph showing a huge ulcerated lesion on the dorsum of left foot.

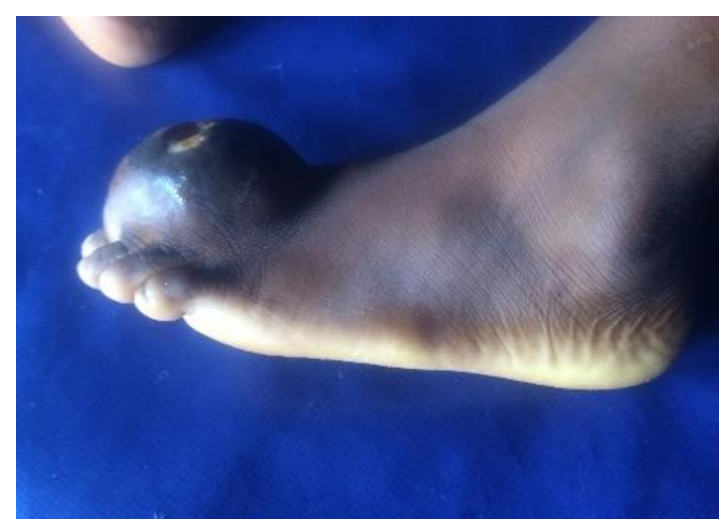

Figure 2: The position of lesion definitely would affect wearing of shoe. 
Examination showed an ulcerated $5 \mathrm{~cm}$ by $4 \mathrm{~cm}$ soft to firm mass on the dorsum of the left foot. It was mildly tender, not attached to the underlying structures but attached to the hyper-pigmented skin around the ulcer (Figures 1 and 2). The ulcer had an undermine edge and does not bleed to touch. There was no slipping sign. The groin nodes were firm and tender. She could move her left toes and she walked unshod with a limp. The chest, abdomen and other systems were normal.

At this point, the clinical diagnosis was unclear and the plan was to do an excision biopsy. Her investigations which included complete blood count and urinalysis were within normal ranges. Her hemoglobin electrophoresis was AA. Plain radiograph of the foot was also normal. Informed consent was obtained and under general anesthesia, and proximal tourniquet, excision biopsy was performed. Intra-operative, the mass was yellowish, shiny, fatty and lobulated with good plane and enucleating it was fairly easy (Figures 3). After care including antibiotics was administered. Histo-pathological examination revealed mature fat cells with empty cytoplasm and eccentric nuclei encased in fibrous capsule but interspersed by fibroblasts and blood vessels without any epithelium which are consistent with lipoma. Patient was discharged after 5 days. By six weeks post-operative follow up, she had fully recovered and was wearing her shoes and had resumed her school activities.

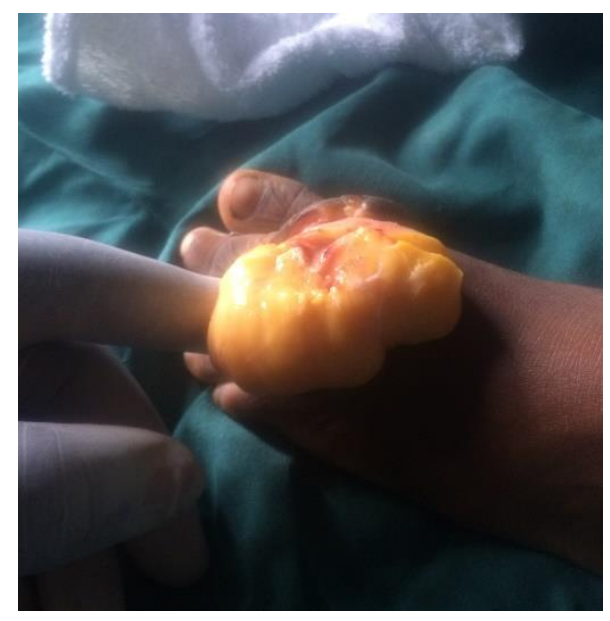

Figure 3: An intraoperative clinical photograph showing easy and complete enucleation of a well encapsulated and lobulated fatty mass.

\section{Discussion}

Lipomas are the commonest form of benign soft tissue tumors consisting of mature fat cells encapsulated in a thin fibrous tissue. These neoplastic lesions are ubiquitous and can occur in any organ of the body that has adipose tissues. Lipomas could occur as solitary or multiple lipomatas. Solitary lipomas are commoner in women in their $5^{\text {th }}$ and $6^{\text {th }}$ decades especially in the obese and consist of $80 \%$ of all lipomas and do not show any familial inheritance $[4,5]$. Multiple lipomatas on the other hand are more common in adult males and occur as lipomatosis with familial predilection, and some variants are autosomal dominant. Some are also symmetrical in distribution and could be associated with Dercum's disease (painful multiple lipomatas\} also known as adiposis dolorosa [6], Madelung's disease (benign symmetric multiple lipomatas in the head and neck and proximal extremities) and Gardner's disease (intestinal polyposis, osteoma) [7]. Whatever type of lipoma, these lesions are very rare in children except for the occasional painful angiolipoma which occur in younger age group than the conventional solitary lipomas [8]. 
Lipomas are slow growing tumors that present as painless, palpable small masses and so are often overlooked by the patients until they get to appreciable sizes [9]. They are mostly subcutaneous but could be sub-fascial or intramuscular and are usually less than $2 \mathrm{~cm}$ but some can reach $20 \mathrm{~cm}$ in their widest diameters [10]. Large tumors could give rise to pressure symptoms on the surrounding structures like nerves or tubular organs resulting in pain. Stretching of the investing structure including the skin can also give rise to pain. In our patient, the location of the tumor on the dorsum of the foot with thin stretched out skin and pressure on the adjacent digital nerves and the ongoing infection could be responsible for the pains she experienced. In the absence of trauma, the possible explanation for the ulceration at the summit of the mass is pressure effect on the skin giving rise to ischemia and necrosis (Figure 1).

The pre-biopsy diagnosis of lipoma in this patient was not definite because of the age of the patient, the unusual location of the tumor in the distal extremity in addition to the ulceration and the absence of the typical slipping sign. The mass was quite superficial and so we did not think ultrasound scan would be extremely beneficial especially as the features of the mass made it amenable to excision. In resource rich environments, magnetic resonance imaging would increase the diagnostic yield but still should be confirmed by biopsy. Lipomas contains in addition to mature fat cell, thin fibrous capsules and this is what distinguishes it from aggregation of fat cells [5] as seen in obese people. In this patient, we relied only on histo-pathological diagnosis to chart a way forward for her treatment which fortunately ended with the complete surgical excision and the routine post-operative care.

The treatment of choice for lipoma is mainly surgical as this is more likely to ensure a complete removal unlike steroid injection that causes fat atrophy or liposuction which usually would leave some fat tissues and the capsules behind [11]. However, the latter methods leave minimal scars. If steroid injections are repeated, skin hypopigmentation may result. Solitary lipoma of a small size could be left alone but if a patient has cosmetic concern based on its' anatomical location, then irrespective of size, the lipoma should be removed. The excision of the mass en bloc in our patient was made possible by the good plane and lack of infiltration of the tumor that is typical of benign lesion. Therefore, avoiding injuries to the digital nerves and the dorsalis pedis artery were possible and the patient recovered without paresthesia or post-operative hematoma. Some authors had reported these complications in addition to fat embolism, seroma, cellulitis, wound infection and wound breakdown in their studies $[1,3,8]$.

The reasons for excising the tumor in our patient were pains, the large size with the attendant cosmetic dysfunction and also importantly to make a definitive diagnosis upon which proper treatments were based. The diagnosis put to rest the worries of the parents concerning the initial clinical diagnosis and the proposed treatment option made by their family clinician. The lesson here is that a solitary lipoma may present differently from the usual pattern. Even when a lesion appears malignant clinically, a definitive statement should only be made after a histo-pathological confirmation and by this way, unnecessary worries will not be placed on the patient and family members like in this index case. 


\section{Conclusion}

It was both psychological and physical relief to confirm that a lesion suspected to be malignant due to its' unusual presentation was a lipoma. The excision was both diagnostic and therapeutic and patient as expected made a full recovery and went back to school within a short period.

\section{Consent}

The patient's parents gave an unconditional approval for this work and the images to be published.

\section{Conflict of interest}

None.

\section{Funding}

The work was self-sponsored.

\section{References}

1. Salam G. Lipoma excision. Am Fam Physician 65 (2002): 901-905

2. Yakubu A, Edino S, Mohammed A. Giant and complicated subcutaneous lipoma of the neck. WAJM 27 (2008): 44-46.

3. Truhan AP, Garden JM, Caro WA, et al. Facial and scalp lipomas: case reports and study of prevalence. J Dermatol Surg Oncol 11 (1985): 981-984.

4. Luba MC, Bangs SA, Mohler AM, et al. Common benign skin tumors. Am Fam Physician 67 (2003): 729738.

5. Kim YH, Reiner L. Ultrastructure of lipoma. Cancer 50 (1982): 102-106.

6. Brody HJ, Meltzer HD, Someren A. Spindle cell lipoma. An unusual dermatologic presentation. Arch Dermatol 114 (1978):1065-1066.

7. Austin R, Mack G. Infiltrating (intramuscular) lipoma and angiolipoma. A clinico-pathologic study of six cases. Arch Surg 115 (1980): 281-284.

8. Kulia V, Kaushal N, Pahwa D. Giant subcutaneous solitary lipoma arising in the neck. Case Report and review of literature. webmedcentral, Maxillofacial Surgery 2 (2011): wmc001838.

9. Del-Agua C, Felipo F. Adenolipoma of the skin. Dermatol online J 10 (2004): 9-15.

10. Shetty N, Shabari UB, Jaydeep NA, et al. Solitary lipoma in the retromandibular region. Indian J Dent 6 (2015): 49-52.

11. Medina C, Schneider S. Giant submental lipoma: case report and review of literature. Can J Plast Surg 15 (2007): 219-222. 